\title{
Q̈FORSETI
}

Forseti. Revista de Derecho. Volumen 9, № 13, Lima, 2021, pp. 21 - 45

\section{Reflexiones en torno al SEIA, a 20 años de su creación}

\author{
Vito Verna*
}

Resumen. - El artículo subraya la naturaleza específica del Estudio de Impacto Ambiental (EIA) y su función dentro del sistema de gestión ambiental; haciendo especial énfasis en la participación ciudadana como instrumento clave para lograr que los EIA sean instrumentos confiables y útiles.

Asimismo, aborda los problemas que enfrenta el SEIA para, una vez construidos y en operación los proyectos de inversión, introducir cambios y mejoras, tanto al proyecto como a los planes de manejo ambiental. Finalmente, se señala las falencias regulatorias que presenta la regulación ambiental transectorial del SEIA cuando se trata de Estudios Ambientales (EA) correctivos.

\begin{abstract}
The article highlights the specific nature of the Environmental Impact Assessment (EIA) and its role within the environmental management system, with special emphasis on citizen participation as a key instrument for making EIAs reliable and useful instruments.

It also addresses the problems faced by the SEIA to introduce changes and improvements, both to the project and to the environmental management plans, once the investment projects have been built and are in operation. Finally, it points out the regulatory shortcomings of the cross-sectoral environmental regulation of the SEIA when it comes to corrective Environmental Studies (EA).

Palabras claves. - Estudio de Impacto Ambiental - Instrumentos de Gestión Ambiental - Regulación Ambiental.
\end{abstract}

Keywords. - Environmental Impact Assessment - Environmental Management Instruments - Environmental Regulation.

* Abogado por la PUCP, Magister en Economía Gestión Ambiental por la Universidad Comercial Luigi Bocconi, socio de CMS Grau y profesor universitario en la PUCP y UPC. Este artículo ha sido elaborado con la muy significativa participación del abogado Mauricio Black, a quien le agradezco mucho el importante aporte a esta, nuestra humilde contribución 


\section{Introducción ${ }^{12}$}

En el momento en que empezaron a aparecer normas jurídicas que regulaban las actividades humanas en función a su interrelación con la naturaleza, no solo apareció el Derecho Ambiental, la ciencia se convirtió en un componente fundamental en la creación e interpretación del derecho positivo ambiental.

Qué actividades debemos incentivar, cuáles restringir, en qué medida la sustancia A, B, o C, disminuye las posibilidades de concebir o aumenta las probabilidades de contraer una enfermedad, cuál es el nivel máximo de concentración de gases efecto invernadero presentes en la atmosfera que no representa una amenaza para la estabilidad climática global, cuáles sustancias químicas amenazan la capa de ozono y qué función desempeña esta capa para la biósfera; estas y miles de interrogantes más deben ser respondidas por las ciencias, y dependiendo de sus respuestas, los países deben negociar acuerdos internacionales y el Derecho Ambiental deberá escribirse, o mejor sea dicho, empezar a ser discutido, consensuado, escrito y aplicado.

Hoy tenemos certezas respecto de las limitaciones que enfrenta la regulación para frenar los daños que seguimos generando a la naturaleza. Estas limitaciones se explican en varios factores, sin embargo, queremos resaltar aquel referido a la incertidumbre, por el cual, resulta imposible anticipar todas las consecuencias que una actividad humana provocará en el sistema ambiental.

En ese escenario de incertidumbre, se forja el Sistema Nacional de Evaluación del Impacto Ambiental (SEIA), que se inspira, no solo en la finalidad de prevenir la afectación negativa del ambiente, sino en la necesidad de ofrecer una respuesta especial, singular y ad hoc para una actividad particular (proyecto) que entrará en interacción con un ecosistema que siempre será único e irrepetible.

Para el profesor Gomez Orea “Todo estudio de impacto ambiental debe plantearse con carácter específico para cada caso; gráficamente se suele decir que debe ser como un traje a la medida ${ }^{3 \prime}$. Asimismo añade que:

“La Evaluación de Impacto Ambiental (EIA) es uno de los principales instrumentos de gestión ambiental, cuya mayor virtualidad la adquiere cuando se utiliza de forma complementaria al resto de los instrumentos...4"

Este artículo pretende resaltar la importancia de la especialidad como criterio principal, fundacional, esencial y finalista del SEIA, y analizarlo en el marco de la

1 Por EIA nos referimos indistintamente a la Declaración de Impacto Ambiental, el Estudio de Impacto Ambiental semi detallado y el Estudio de Impacto Ambiental detallado.

2 Por EA nos referimos conjuntamente a los instrumentos de gestión ambiental complementarios al SEIA y a los EIA.

3 GOMEZ OREA, Domingo. Evaluación de Impacto Ambiental. Un instrumento preventivo para la gestión ambiental. Segunda edición. Madrid: Ediciones Mundi-Prensa, 2002, p 285.

4 GOMEZ OREA, Domingo. Evaluación de Impacto Ambiental. Un instrumento preventivo para la gestión ambiental. Segunda edición. Madrid: Ediciones Mundi-Prensa, 2002, p. 217 
simplificación administrativa, la cual podría desfigurarlo a través de la estandarización o simplificación de los EIA.

La enorme diversidad de variables que engloba un ecosistema, en el orden biológico, físico y químico, las cuales entrarán en contacto con los componentes de un proyecto, deben ser analizados en clave sistémica, para identificar la posible o probable aparición de impactos ambientales o sociales que deberán ser gestionados para potenciarlos, prevenirlos, mitigarlos o compensarlos.

Estos impactos al ser una res nova no pueden ser anticipados por ninguna norma legal general y, por lo tanto, requieren de un abordaje especial, el cual es atendido a través del EIA y el plan de manejo ambiental que este documento contiene.

En este orden de ideas, la evaluación del impacto ambiental y el EIA constituyen instrumentos ambientales dirigidos a ofrecer una alternativa a la necesaria especialidad que demanda la gestión ambiental.

Una segunda reflexión que compartimos en las líneas que siguen, está inspirada en la tensión que plantea un SEIA en el que predomina notoriamente un enfoque preventivo en desmedro de otro preventivo-adaptativo.

Desde nuestra visión, el SEIA que nos rige actualmente presenta deficiencias para, una vez construidos y en operación los proyectos, el administrado y el Estado puedan adaptar a lo largo del tiempo las medidas del plan de manejo ambiental a la realidad. En definitiva, es esta realidad la que nos presenta los impactos concretos y palpables, reales y apremiantes.

En este sentido, creemos que el SEIA debe considerar con igual intensidad las dos oportunidades que tiene para gestionar adecuadamente los impactos ambientales; la primera, que aparece en el momento en que diseñamos y evaluamos los proyectos (predictivo y anticipativo); $y$, la segunda, que se presenta una vez iniciada la etapa de construcción y operación del proyecto, durante la cual, la realidad de los acontecimientos mitiga la incertidumbre y nos permite hacer lo necesario para conservar el ambiente y la paz social.

En tal sentido, el presente artículo plantea la urgente necesidad de iniciar la búsqueda de respuestas a dos preguntas, que entendemos centrales para nuestro SEIA:

i. ¿El SEIA, tal y como es regulado hoy, constituye un sistema que busca soluciones ambientales especiales acordes a la particularidad de los proyectos y ecosistemas en interacción?

ii. ¿El SEIA es un sistema que permite la corrección preventiva, continua y adaptativa, de las desviaciones entre los impactos estimados y los impactos reales? 
Mas que dos preguntas a las cuales debe buscarse una respuesta, estas son dos cuestiones que deben servir como lineamientos tanto para la elaboración de EIA como para su evaluación.

Creemos que especialidad y corrección adaptativa deben ser siempre dos características reconocibles en los EIA para lograr la eficacia de este instrumento de gestión ambiental, por las razones que pasamos a desarrollar.

\section{La especialidad del EIA}

La regulación ambiental cuenta con disposiciones de protección ambiental generales, aplicables de forma homogénea a un amplio espectro de entornos ambientales, estas normas, por su aplicación extendida y estandarizada son fáciles de comprender y fiscalizar, sin embargo, en algunos escenarios podrían demostrar ser insuficientes o excesivas al momento de prevenir, mitigar o compensar un impacto ambiental.

Ejemplos emblemáticos de este tipo de normas de aplicación general, lo constituyen los Límites Máximos Permisibles (LMP), los Estándares de Calidad Ambiental (ECA) o normas sobre residuos sólidos que, en el primer caso, fijan en forma general un límite a la contaminación que puede ser liberada al ambiente legalmente, y en el segundo, se establecen parámetros para evaluar el riesgo ambiental que podría estar presente en alguno de sus componentes. En ningún caso, estas normas responden a la realidad (biológica, física u química) de los escenarios en que se aplicarán.

Este tipo de regulación general cumple una función muy importante para la gestión ambiental, debido a un gran número de importantes razones que no es momento de abordar, sin embargo, estas normas requieren ser complementadas o sustituidas con medidas especiales, diseñadas a la medida de los impactos ambientales concretos identificados.

Este tipo de medidas especiales, cuando se encuentran en los EA reciben el nombre de compromisos ambientales. Los compromisos ambientales, nacen de un EA, que puede ser preventivo (Declaración de Impacto Ambiental o EIA), o complementario al SEIA (Programas de Adecuación Ambiental, Planes de cierre, etc.) y tienen como característica: i) su especialidad (han sido concebidos y diseñados para atender uno o más impactos ambientales concretos), ii) tienen fin ambiental (y/o social) buscan prevenir, mitigar o compensar un impacto ambiental determinado, y iii) fuente meta o extra regulatoria, es decir, reconocen como única fuente de creación un EA, no son creados por normas jurídicas; de esta forma se mantiene el rol complementario respecto de la regulación ambiental general.

El ecosistema es complejo, al igual que las comunidades humanas asentadas en él, por lo tanto, las medidas de gestión ambiental que un proyecto debe cumplir para lograr una integración armónica con su entorno social y ambiental deben ser 
especiales en cada caso; recetas estandarizas aplicadas a situaciones altamente complejas podrían ser contraproducentes.

El valor de la especialidad radica en su capacidad para responder adecuadamente a diversas situaciones, cada una distinta de la otra. Imaginemos, por ejemplo, que un proyecto descargará efluentes en un cuerpo de agua léntico que alberga una gran cantidad de especies de flora y fauna, algunas de ellas endémicas; en ese caso, debería estudiarse científicamente los posibles efectos de los compuestos químicos presentes en este efluente en la flora y fauna presente en el lago, ello con la finalidad de establecer si existe la posibilidad de que se produzcan impactos ambientales significativos, y, de ser este el caso, adoptar medidas apropiada a este caso en particular. Estas medidas podrían involucrar compromisos relativos a monitoreos frecuentes y la aplicación de restricción cualitativas y cuantitativas a sus efluentes, que podrían ser más altos que los establecidos en los LMP aplicables al caso.

Sólo el EA puede tomar en consideración la presencia y vulnerabilidad de las especies endémicas que presentamos en el ejemplo, y establecer las medidas necesarias para su protección. La especialidad del EA constituye la respuesta a la complejidad que plantea el proyecto y el entorno, y en ella, precisamente, radica su valor como instrumento de gestión ambiental complementario de la regulación general.

El precio de la especialidad se paga en tiempo. En efecto, la observación y registro de los ciclos naturales, el estudio de las especies y de las condiciones físicoquímicas, los procesos de participación ciudadana, el relacionamiento con comunidades culturalmente distintas, el análisis de la información, el proceso de evaluación del EIA, todo ello y más, consume tiempo. A mayor detalle o especificidad de la información, por lo menos en nuestro país, corresponde mayor tiempo de elaboración y evaluación del EIA.

El EIA constituye la respuesta que ofrece el Derecho Ambiental a la complejidad del ecosistema, a través de este estudio debemos caracterizar el entorno: la biosfera (flora y fauna presentes), geosfera (clima, suelo, agua y aire) y el medio social (población, composición, salud, educación, economía, hábitos, temores, intereses, etc.); luego debemos describir el proyecto, acto seguido, identificar, a partir de la interrelación (ambiente - proyecto), los impactos ambiental y sociales, valorarlos y cualificarlos, para finalmente, diseñar las medidas especiales (compromisos) para prevenir, mitigar o compensar los impactos.

Ahora bien, en este punto aparece una pregunta clave: ¿Hasta qué punto debemos profundizar o detallar el estudio del ecosistema social y ambiental y sus interacciones con el proyecto?

El Derecho Administrativo cobija las normas que regulan el procedimiento de elaboración y evaluación del EIA, y, en consecuencia, una parte de la respuesta a esta cuestión la debemos buscar en los derechos y garantías reconocidos por esta 
rama del Derecho a los administrados, tales como: "plazo razonable", o en principios como el de "Simplicidad".

En este orden de ideas, es claro que nos situamos frente a dos fuerzas en aparente oposición, por un lado, tenemos la necesidad de producir EIA especiales y adecuados a cada ecosistema y proyecto: y por el otro, tenemos los derechos y principios del Derecho Administrativo, los cuales buscan diseñar procedimientos razonablemente simples y céleres.

Ahora bien, ¿Cómo es que la complejidad humana, social y ambiental ingresan al EIA y se procesa para, a partir de ese punto, producir planes de manejo ambiental especiales, eficientes y eficaces? Creemos que las vías a través de las cuales se introduce información específica, relevante y singular a los EIA son: i) los procesos de participación ciudadana; $y$, ii) el proceso de construcción de la línea base ambiental.

En qué forma la Ley y Reglamento del SEIA trata ambos procesos constituye un dato importante, aunque no definitivo, para poder ensayar una opinión sobre las probabilidades que el EIA sea un instrumento verdaderamente complementario a los demás instrumentos de gestión ambiental (IGA) y especialmente diseñado para un proyecto particular y específico.

\section{i. Participación ciudadana en la regulación}

La Participación Ciudadana aparece en el artículo 1 de la Ley del SEIA, cuando se listan las tres finalidades de la Ley: “c) El establecimiento de los mecanismos que aseguren la participación ciudadana en el proceso de evaluación de impacto ambiental5"; y, en el Capítulo III de la mencionada Ley.

En el Capítulo III, la Ley consagra la necesidad de promover la participación ciudadana, tanto en el proceso de elaboración de EIA (instancias no formales) como en el proceso de evaluación del EIA (instancias formales), siendo, por lo tanto, un deber del proponente y del Estado lograr asegurar la participación efectiva de la población, incorporar su voz y que ésta tenga un valor relevante y notorio en ambos procesos.

La Ley establece, respecto de la autoridad evaluadora: i) la facultad de solicitar información a la ciudadanía durante la etapa de evaluación del EIA, (facultad no desarrollada) ii) la obligación de realizar una "consulta formal" respecto del proyecto en evaluación, y iii) que la autoridad ponga a disposición de la ciudadanía el texto del EIA y difunda su existencia a través de medios convencionales y electrónicos de comunicación ${ }^{6}$.

5 Ley N²7446. Ley del Sistema Nacional de Evaluación del Impacto Ambiental (SEIA). Diario Oficial El Peruano. Lima, Perú. 23 de abril de 2001.

$6 \quad$ Ley N ${ }^{\circ}$ 27446. Ley del Sistema Nacional de Evaluación del Impacto Ambiental (SEIA). Diario Oficial El Peruano. Lima, Perú. 23 de abril de 2001. Artículo 14. 
Asimismo, se identifica a la Audiencia Pública como un mecanismo de participación ciudadana para los EIA.

Por su parte, el proponente debe presentar a la autoridad su Plan de Participación Ciudadana y sufragar los gastos que demande todas las acciones ahí consideradas, desde la difusión del EIA hasta la audiencia pública.

A su turno, el Reglamento del SEIA, en el artículo 68, define a la participación ciudadana como:

“...un proceso dinámico, flexible e inclusivo, que se sustenta en la aplicación de múltiples modalidades y mecanismos orientados al intercambio amplio de información, la consulta, el diálogo, la construcción de consensos, la mejora de proyectos y las decisiones en general, para contribuir al diseño y desarrollo responsable y sostenible de los proyectos de inversión, así como de las políticas, planes y programas de las entidades del sector público ${ }^{7 \prime}$.

Y establece su obligatoriedad en todos los tipos de EIA, incluso cuando se trate de una Declaración de Impacto Ambiental (DIA).

El artículo 70 define los mecanismos de participación ciudadana como:

“...instrumentos para la difusión de información y la generación de espacios para la formulación de opiniones, observaciones, sugerencias, comentarios y otros aportes orientados a mejorar los procesos de toma de decisiones respecto de los estudios ambientales... $8^{\prime \prime}$

Asimismo, proporciona un listado referencial de los mecanismos, entre los cuales tenemos los siguientes:

“...publicación de avisos; distribución de Resúmenes Ejecutivos y acceso público al texto completo del estudio ambiental; buzones de observaciones y sugerencias; oficinas de información y participación ciudadana; visitas guiadas; consulta con promotores; mecanismos para canalizar observaciones y sugerencias ante la autoridad; talleres o reuniones informativas; audiencias públicas con participación de intérpretes en lenguas locales...9"

Parte importante de la regulación sobre participación ciudadana aplicable a los procesos de elaboración y evaluación de los EA se encuentra en el Reglamento sobre Transparencia, Acceso a la Información Pública Ambiental y Participación y Consulta Ciudadana en Asuntos Ambientales, aprobado por D.S. 002-2009MINAM, el cual es de aplicación a los organismos adscritos al Ministerio del

7 Decreto Supremo No 019-2009-MINAM. Reglamento de la Ley del Sistema Nacional de Evaluación de Impacto Ambiental. Diario Oficial El Peruano. Lima, Perú. 25 de setiembre de 2009. Artículo 68.

8 Decreto Supremo N 019-2009-MINAM. Reglamento de la Ley del Sistema Nacional de Evaluación de Impacto Ambiental. Diario Oficial El Peruano. Lima, Perú. 25 de setiembre de 2009. Artículo 70.

9 Ídem. 
Ambiente, así como a las entidades integrantes del Sistema Nacional de Gestión Ambiental, en los tres niveles de gobierno.

Este dispositivo contiene normas que desarrollan: i) el acceso a la información ambiental, ii) los mecanismos para transparentan la información con la que cuentan las instituciones públicas, iii) la participación ciudadana y sus mecanismos, primero en términos generales y luego, específicamente, cuando se trata de la participación ciudadana en el marco de los procesos de elaboración y evaluación del impacto ambiental.

En este último extremo, el reglamento desarrolla, en modo, forma y oportunidad dos mecanismos clave en los procesos de elaboración y evaluación de impacto ambiental, a saber: el Taller Participativo y la Audiencia Pública.

El Reglamento encarga al proponente la elaboración del plan de consulta, documento que se expresa en el Plan de Participación Ciudadana, el cual es sometido a evaluación de la autoridad pública, sin embargo, ello nunca será suficiente para generar confianza en el proceso, requisito indispensable para que la población exprese la información que conoce, los sentimientos que experimenta y las observaciones que tiene.

En tal sentido, el Reglamento adolece, en este extremo, de las normas que aseguren el involucramiento activo, cercano y confiable de los funcionarios públicos con el proceso, asignándoles, deberes, actuaciones y resultados específicos, que aseguren la participación de la población y la sociedad civil organizada, en especial de las instituciones académicas, gremios profesionales y asociaciones empresariales más cercanas al proyecto.

Respecto de los reglamentos de protección ambiental sectoriales, el minero, aprobado por D.S. 040-2014-EM ${ }^{10}$, constituye una buena fuente de consulta, por el interesante desarrollado del tema de participación ciudadana y relacionamiento comunitario que ofrece.

En primer lugar, el reglamento minero contempla el requisito de participación ciudadana temprana y obligatoria en el artículo 29, al imponer al proponente la obligación de comunicar a la autoridad ambiental la fecha de inicio de elaboración del EIA o de su modificación, indicando los mecanismos de participación ciudadana efectuados antes de la elaboración del estudio ambiental, asimismo, se establece que el Plan de Participación Ciudadana se presenta una vez concluida la elaboración de la línea base del estudio y antes de la presentación del EIA.

10 Decreto Supremo Nº40-2014-EM. Reglamento de Protección y Gestión Ambiental para las Actividades de Explotación, Beneficio, Labor General, Transporte y Almacenamiento Minero. Diario Oficial El Peruano. Lima, Perú. 12 de noviembre de 2014. 
Respecto de la actuación e involucramiento de los funcionarios públicos en el proceso, el artículo 29 establece una norma central, la cual acertadamente prescribe que:

“c) La línea base, la identificación y evaluación de los impactos, así como la estrategia de manejo ambiental, debe ser elaborado por la consultora, en coordinación con la Autoridad Ambiental Competente y con participación del titular minero...11"

De esta forma se pretende involucrar, desde instancias iniciales, a la autoridad de certificación en el proceso de elaboración del EIA, la cual deberá asegurarse que la metodología propuesta por el proponente asegure entre otras cosas, la participación ciudadana de las personas interesadas, haciendo especial énfasis en aquellas que serán afectadas por el proyecto.

En el artículo 122, se regula la visita de campo para el levantamiento de la Línea Base, la cual puede ser solicitada por el titular de la actividad minera, antes de presentado el estudio $u$ ordenada por la autoridad ambiental dentro de una ventana de oportunidad, que va desde la comunicación de inicio de elaboración del EIA hasta 30 días posteriores a la realización de la última audiencia. La visita busca dar a conocer en el sitio, los alcances generales del EIA, destacando los aspectos más relevantes identificados en el proceso de elaboración del estudio.

La autoridad ambiental competente podrá convocar la participación de las autoridades y población. Además del proponente o su representante, deberán asistir la consultora encargada de realizar el estudio.

\section{ii. La Línea de Base en la regulación}

Respecto de la Línea de Base, la Ley de SEIA se limita a establecer algunos permisos burocráticos que deberá tramitar y obtener el proponente del proyecto a efecto de poder iniciar con la elaboración del referido estudio.

Por su parte, el Reglamento de la Ley del SEIA no dedica ninguna norma al tratamiento de la línea de base. Las menciones a la línea base se encuentran en los anexos de esta norma (Anexo 1: Definiciones y Anexos 3 y 4, sobre Términos de Referencia de EIA), con lo cual queda bastante claro que, en su momento, el estudio de línea base fue concebido como un tema técnico de responsabilidad exclusiva del proponente y consultora ambiental.

Por Ley 30327, “Ley de Promoción de las Inversiones para el Crecimiento Económico y el Desarrollo Sostenible", del mes de mayo de 2015, se introdujo la posibilidad del uso libre y compartido de la información de la línea base de un EIA

11 Decreto Supremo Nº40-2014-EM. Reglamento de Protección y Gestión Ambiental para las Actividades de Explotación, Beneficio, Labor General, Transporte y Almacenamiento Minero. Diario Oficial El Peruano. Lima, Perú. 12 de noviembre de 2014, artículo 29. 
aprobado, ya sea para el mismo proponente o un tercero, el cual puede pertenecer al mismo sector económico o no.

Las condiciones para el uso compartido de la información de la línea de base son: que no haya transcurrido más de 5 años desde la aprobación del EIA, y que el nuevo proyecto de inversión se encuentre íntegramente ubicado en el área física de la línea base preexistente.

El uso parcial de una línea base preexistente se admite igualmente, sin embargo, requiere la conformidad de la autoridad competente, respecto del espacio geográfico que no ha sido materia de la línea base preexistente.

Estas disposiciones fueron desarrolladas por un reglamento ${ }^{12}$, en el que se precisan temas como que el uso compartido de la línea de base aplica también para la elaboración de modificaciones de los EIA y/o instrumentos de gestión ambiental complementarios; se establecen prerrogativas para las autoridades de certificación ${ }^{13}$, deberes del proponente del proyecto ${ }^{14}$, el proceso de supervisión y acompañamiento en campo que realiza la autoridad de certificación al proponente y consultora ambiental mientras elabora la línea de base ${ }^{15}$ y el informe final resultante de la supervisión antes mencionada ${ }^{16}$.

12 Decreto Supremo Nº05-2016-MINAM. Reglamento del Título II de la Ley de Promoción de las Inversiones para el Crecimiento Económico y el Desarrollo Sostenible y otras medidas para optimizar y fortalecer el Sistema Nacional de Evaluación del Impacto Ambiental. Diario Oficial El Peruano. Lima, Perú. 19 de julio de 2016.

13 La autoridad de certificación puede solicitar al proponente que se complemente la información de la línea base preexistente cuando la información de la misma no sea compatible con la información necesaria para el nuevo EA; cuando el ecosistema hubiera variado; cuando la línea base preexistente no cubra toda la información necesaria o no tenga el nivel de profundidad y detalle necesario, entre otros supuestos.

El Reglamento faculta a la autoridad de certificación a convocar reuniones de trabajo con el titular y entidades públicas a fin de tratar aspectos técnicos especializados, solicitar información de acuerdo con el Plan de Trabajo de elaboración de la línea del base presentado por el proponente y realizar visitas de campo, entre otras.

14 a) Presentar el Plan de Trabajo para la elaboración de la línea base del EIA; b) Comunicar periódicamente, el cumplimiento de las actividades de acuerdo al Plan de Trabajo; así como los cambios que pudiera necesitar y que impliquen nuevos aspectos a ser supervisados; c) Permitir y facilitar el acceso al área de levantamiento de la línea base, para el desarrollo de las visitas de campo por parte de la autoridad; Proporcionar la información y documentación solicitada; tomar en cuenta las recomendaciones realizadas por la autoridad, entre otras.

15 La supervisión o acompañamiento de la autoridad en la elaboración de la línea de base, cuenta con un procedimiento, el cual inicia una vez que el proponente comunica el inicio de la elaboración del EIA, incluyendo el Plan de Trabajo para la elaboración de la línea base, el cual debe contener los aspectos relacionados con el levantamiento de información en campo y gabinete, previo a su ejecución; la autoridad, convoca al titular, a los opinantes técnicos y a las entidades autoritativas a reuniones de coordinación, a fin de conocer los alcances generales del proyecto, el Plan de Trabajo, los aspectos técnicos especializados y los posibles riesgos ambientales que pudieran identificarse en el área donde se desarrolla el proyecto; en atención a las características del proyecto y a la información obtenida en las reuniones de trabajo, la autoridad competente comunica al titular la decisión de realizar visitas de campo para supervisar in situ la elaboración de la línea base (Artículo 39).

16 Luego de la supervisión se emite el informe de supervisión de la elaboración de la línea base del EIA, describiendo las actuaciones y hallazgos relevantes, así como las conclusiones y recomendaciones formuladas por los opinantes técnicos y las Entidades Autoritativas, 
El reglamento minero también dispensa un tratamiento extenso a la línea de base, concepto que menciona en 24 oportunidades, y que es objeto de definición, normas sobre elaboración conjunta entre consultoría, proponente y Estado, la necesidad de promover la participación ciudadana en su elaboración, sobre su actualización, etc.

Por su parte, el Ministerio del Ambiente aprobó, por RM 455-2018-MINAM, la Guía para la Elaboración de la Línea Base y la Guía para la identificación y caracterización de impactos ambientales, documento de carácter técnico orientador, que resalta la real importancia de este estudio para el EIA.

\section{iii. Reflexión General}

El SEIA consta de una Ley y un Reglamento, ambos dispositivos de aplicación transectorial y subnacional; y, a continuación, de un conjunto de reglamentos sectoriales y subnacionales de desarrollo ulterior, los cuales buscan adaptar la regulación general a las particularidades sectoriales y subnacionales, pero sin perder la debida coherencia con la Ley y Reglamento del SEIA.

En este orden de ideas, las disposiciones de la Ley 30327 y su Reglamento deberían ser incorporadas, en lo que sea pertinente, al nivel regulatorio transectorial del SEIA (Ley y Reglamento del SEIA), de forma tal de poder restablecer el diseño regulatorio primigenio de este sistema.

Respecto del tiempo, introducido a esta reflexión como el costo de la especialidad del EIA, creemos que es función de, para el caso de la participación ciudadana, del nivel de confianza presente en la población, y, para el caso de la línea base, de la cantidad y calidad de la información social y ambiental disponible para el proponente antes de emprender el estudio de línea base.

En ambos casos, las variables mencionadas (confianza e información) son inversamente proporcionales al tiempo, a menor confianza corresponde mayor tiempo y a menor cantidad y calidad de información sobre el territorio y la sociedad corresponde también mayor tiempo de trabajo y análisis.

En el Perú, adolecemos de ambas cosas, confianza e información y, por lo tanto, el mayor tiempo que en el Perú demandan los proceso relacionados con el EA no necesariamente son debidos completamente a trámites burocráticos irrazonables y/o a funcionarios displicentes e insensibles.

\section{a) Participación Ciudadana}

Sin participación ciudadana no existen posibilidades reales que el instrumento de gestión ambiental (IGA) sea concebido a la medida de una particular realidad

adjuntando copia de las actas de supervisión, el informe es enviado al titular y a las entidades que participaron (Artículo 40). 
social y ambiental, por lo tanto, en atención al criterio o principio de Especialidad del SEIA, debe establecerse con mayor claridad la necesidad de asegurar la efectiva participación ciudadana en la elaboración y evaluación de los EIA.

Creemos que la información que necesita el EIA para delimitar su alcance, identificar los impactos ambientales y sociales, valorarlos correctamente y establecer una estrategia ambiental específica y socialmente aceptable; se encuentra en gran medida en la población y en las instituciones locales, y que el problema principal que enfrentamos radica en conseguir que esta información sea exteriorizada, comprendida por las autoridades, incorporada en el EIA y tomada en cuenta.

Luego de prácticamente 20 años de vigencia de la Ley del SEIA, lo establecido en su artículo 1 sigue siendo acertado, la finalidad es "asegurar la participación ciudadana" sin embargo, el desarrollo regulatorio contenido en la Ley y Reglamento del SEIA es aún parcial y no establece con claridad los mecanismos y procedimientos que debemos emprender, en particular desde el Estado, para lograr una efectiva participación ciudadana.

El “Acuerdo Regional sobre el Acceso a la Información, la participación Pública y el Acceso a la Justicia en Asuntos Ambientales en América Latina y el Caribe" (En adelante Acuerdo de Escazú), actualmente abierto a ratificaciones para los países de la región17, establece una participación abierta e inclusiva (Art. 7) la cual debe promoverse desde etapas bastante tempranas o preliminares, en este caso de los proyectos (Art. 7 Numeral 4), garantizando a los ciudadanos un plazo razonable para entender la información ambiental (Art. 7 Numeral 5), la cual debe ser presentada en modo y forma comprensible, obviamente teniendo como referencia a los receptores de la información, quienes podrían ser culturalmente distintos a los emisores del mensaje (Art. 7 Numeral 6), el acceso a medios apropiados para presentar sus observaciones $y$, muy importante, que la autoridad acredite, primero que ha tomado en cuenta las observaciones de la población y, en qué modo han sido consideradas en su decisión (Art. 7 Numeral 7).

Respecto a los Proyectos que se prevén tendrán impactos ambientales significativos, el acuerdo de Escazú, prescribe (Art. 7 Numeral 16) que la autoridad pública:

“... realizará esfuerzos por identificar al público directamente afectado por proyectos y actividades que tengan o puedan tener un impacto significativo

17 El Congreso, a través de su Comisión de Relaciones Exteriores desestimo la ratificación del Acuerdo de Escazú en el mes de octubre de 2020, por lo que sus disposiciones, por el momento, no tendrán efecto vinculante en nuestro país, aunque sí deberán ser siempre tomadas en consideración para mejorar y porque no interpretar nuestra regulación interna. 
sobre el medio ambiente, y promoverá acciones específicas para facilitar su participación ${ }^{18}$.

Asimismo, establece (Art. 7 Numeral 17) la publicidad del área de influencia del proyecto, las características del mismo, la descripción de los impactos ambientales y las medidas para enfrentarlos, las opiniones técnicas de las autoridades públicas que han opinados, durante el proceso de evaluación ambiental, y finalmente, publicitar las acciones de monitoreo ambiental establecidas.

Rescatamos y subrayamos de las citas efectuadas arriba de Escazú, para efectos de la presente contribución, la importancia de establecer claramente, tanto en la Ley como en Reglamento del SEIA la oportunidad en que debe empezar la participación ciudadana, cual es: antes de iniciar la elaboración del EA; también queremos señalar el rol activo que reconoce el Acuerdo al Estado, el cual debe realizar lo esfuerzos necesarios para: identificar al público directamente afectado, y promover acciones específicas para facilitar su participación.

En línea con el rol del Estado, destacamos la recomendación de la Sociedad Peruana de Derecho Ambiental (SPDA) relativa a que:

“Antes del inicio de los procesos de participación ciudadana se debe dar conocer su objetivo, a efectos de que la población tenga en claro que la implementación de los mecanismos (...) es una oportunidad para obtener información sobre la ejecución del mismo (proyecto), así como dar a conocer las preocupaciones y observaciones con relación al proyecto ${ }^{19}$.

Coincidimos con la SPDA en que, considerando nuestra realidad social, resulta muy importante un trabajo previo, que debería estar a cargo de un actor tercero al proponente y la consultora contratada, que consiste en capacitar y sensibilizar a la población sobre la importancia de su participación efectiva y sobre el funcionamiento y la finalidad de los mecanismos de participación ciudadana.

La participación ciudadana constituye un insumo indispensable del SEIA, por lo tanto, debe ser incorporada en lo necesario a sus normas, creemos en clave de obligaciones de resultados en la ley, considerando sistemas objetivos de medición (indicadores cualitativos y cuantitativos) y como obligaciones concretas de actuación estatal en el Reglamento.

\section{b) Línea de Base}

Si bien en la presente contribución hemos tratado como canales separados de flujo de información a la participación ciudadana y al estudio de línea de base, es un

18 Acuerdo Regional sobre el Acceso a la Información, la participación Pública y el Acceso a la Justicia en Asuntos Ambientales en América Latina y el Caribe. https://repositorio.cepal.org/bitstream/handle/11362/43595/1/S1800429_es.pdf

19 CALLE, Isabel y RYAN, Daniel (coords). La participación ciudadana en los procesos de Evaluación de Impacto Ambiental: análisis de casos en 6 países de Latinoamérica. Lima: SPDA, 2016. 
hecho que la primera nutre de información al segundo, y constituye un factor clave para su calidad.

El otro elemento central para la línea de base es la información social y ambiental que debería existir sistematizada en fuentes de acceso público. Ante esta carencia, se habilitó una medida que podría ser calificada como reactiva o provisional, que consiste en el reúso de líneas de base de otros proyectos.

Rescatamos de la regulación sobre línea de base el énfasis que pone en la actuación del Estado, reservando para él facultades de evaluación, supervisión y acompañamiento, coordinación de instituciones públicas y observación, entre otras, las cuales son necesarias para asegurar que la línea de base contenga la información necesaria para producir un EIA especial y relevante.

En tal sentido, y en concordancia con la necesidad de generar confianza, la autoridad de certificación debería tener un rol más intenso y definitorio en la generación de información social, como actores institucionales y sociales involucrados, relación de la población con los recursos naturales, identificación de percepciones, intereses, expectativas y temores sociales, respecto del proyecto y la definición del área de influencia directa e indirecta del proyecto.

La Guía para la elaboración de la línea base considera a este estudio como:

“... una de las principales herramientas en el proceso de elaboración de los estudios ambientales y constituye los cimientos para realizar la evaluación de los impactos, diseñar las medidas de manejo y hacer seguimiento a la eficacia de las medidas de control propuestas. Por ello la línea base debe ser preparada con un fundamento técnico sólido20".

En tal sentido, la Ley y Reglamento del SEIA deberían incorporar normas que reconozcan la importancia de la información contenida en la línea de base, la definición del concepto, los estándares de calidad que se espera de ella, el rol del Estado en su construcción y los medios a disposición de las autoridades para lograrlo.

\section{Estimación y valoración de impactos ambientales aplicada en la etapa operativa de los proyectos}

El SEIA nace, para el caso de los proyectos de inversión, como un sistema para la prevención y corrección anticipada de los impactos que teóricamente se producirán con ocasión de la construcción y operación de estos proyectos.

Consideramos que, naturalmente, la Ley y Reglamento del SEIA fueron diseñados en torno a la estimación y valoración de impactos teóricos, desarrollándose para

20 Ministerio del Ambiente. Guía para la Elaboración de la línea de base, en el marco del Sistema Nacional de Evaluación del Impacto Ambiental. 2018, p. 5. http://www.minam.gob.pe/wpcontent/uploads/2018/10/Guia-Linea-Base.pdf 
tales efectos: procedimientos administrativos, términos de referencia, criterios de evaluación y guías metodológicas, entre otros documentos; sin embargo, la estimación y valoración de impactos ambientales no termina cuando se emite la certificación ambiental, esta actividad perdura, aunque con diferencias, durante toda la vida útil del instrumento de gestión ambiental.

La actividad estimativa debe permanecer para: i) evaluar el plan de manejo ambiental y mejorarlo de forma permanente; evaluar los impactos derivados de las redefiniciones que, a lo largo de su vida, tendrá el proyecto; y, iii) aprobar y adaptar los denominados instrumentos ambientales complementarios. No obstante ello, la Ley y Reglamento del SEIA debido a su diseño primigenio, no contiene la regulación transectorial necesaria para ordenar la actividad estimativa de proyectos y actividades reales y operativos.

La situación que describimos, caracterizada por vacíos o falta de claridad en la regulación contenida en la Ley y Reglamento del SEIA, ha provocado que los reglamentos sectoriales ambientales avancen en llenar los vacíos y aclarar los conceptos, lo que ha ocasionado que se adopten criterios disímiles y regulación diversa para temas comunes en cada sector productivo, lo que produce desorden en el sistema.

Creemos que el SEIA debe avanzar en desplegar regulación transectorial para las etapas de construcción y operación de proyectos, sin perjuicio que la regulación sectorial continúe con su tarea, la cual consiste en adaptar la regulación general a las particularidades sectoriales.

A continuación, nos referiremos a algunos casos que ejemplifican la problemática descrita:

\section{i. Actualización y Modificación del EA}

Cuando se elabora y evalúa un EIA la estimación de impactos es teórica, se realiza sobre la base de información del proyecto definida a nivel de factibilidad e información social y ambiental del área de interés para el proyecto, obtenida de fuentes primarias y secundarias.

Cuando el proyecto inicia su etapa de construcción y operación podríamos considerar que, al enfrentarnos a impactos reales, la estimación no resulta aplicable; sin embargo, la actividad prospectiva de continuar debido principalmente, a que las interacciones y reacciones entre el proyecto y el entorno continuará mientras ambas entidades coexistan.

¿Cómo las medidas del plan de manejo ambiental responden y se adaptan a un ambiente y proyecto vivo, en constante cambio e interacción? El SEIA, tal y como se encuentra regulado hoy no ofrece una respuesta clara.

\section{a) La actualización del EA}


En el SEIA subsiste a la fecha una notoria falta de uniformidad de criterios al intentar definir la actualización de instrumentos de gestión ambiental. Aun con reglas delimitadas en la normativa de SEIA, al evocar el término, muchos operadores técnicos y legales incluyen erróneamente dentro de sus límites elementos propios de otros instrumentos, como por ejemplo el potencial para proponer la regularización de componentes no evaluados previamente - que es una tarea reservada a los instrumentos correctivos extraordinarios, a los cuales nos referiremos más adelante.

El Reglamento del SEIA regula la actualización de los EIA en su artículo 28 y 30, textos que pasamos a analizar:

Artículo 28:

“( ...)

Las medidas y planes de los estudios ambientales de Categoría I, II y III, están sujetos a actualización cada vez que se realicen cambios o modificaciones que varíen de manera significativa el alcance o posibles impactos del proyecto de inversión materia del estudio ambiental aprobado o en caso que se aprueben nuevas normas que así lo determinen.

La modificación del estudio ambiental o la aprobación de instrumentos de gestión ambiental complementarios, implica necesariamente y según corresponda, la actualización de los planes originalmente aprobados al emitirse la Certificación Ambiental21".

Artículo 30:

"El Estudio Ambiental aprobado, debe ser actualizado por el titular en aquellos componentes que lo requieran, al quinto año de iniciada la ejecución del proyecto y por periodos consecutivos y similares, debiendo precisarse sus contenidos, así como las eventuales modificaciones de los planes señalados en el artículo precedente. Dicha actualización será remitida por el titular a la Autoridad Competente para que ésta la procese y utilice durante las acciones de vigilancia y control de los compromisos ambientales asumidos en los estudios ambientales aprobados $(. . .)^{22 \prime \prime}$.

La actualización, tal y como es regulada fue diseñada para atender la necesidad de sistematización del EIA luego que se produce un cambio o modificación que varíen los impactos del proyecto.

21 Decreto Supremo No 019-2009-MINAM. Reglamento de la Ley del Sistema Nacional de Evaluación de Impacto Ambiental. Diario Oficial El Peruano. Lima, Perú. 25 de setiembre de 2009. 22 Ídem. 
Una primera cuestión consiste en determinar si la actualización se realiza respecto de proyectos en operación con EIA aprobados o es aplicable también a proyecto con EIA aprobados que aún no entran en etapa de construcción y operación.

Como sabemos, todo cambio que involucre una variación de los impactos debe tramitarse a través de un procedimiento de modificación de EIA, el cual puede iniciarse con la presentación de un Informe Técnico Sustentatorio (ITS) -si se trata de cambios que se estima tengan impactos no significativos- o una Modificación de EIA, cuando los impactos previsto han sido valorados como significativos.

La modificación del EIA puede presentarse, luego de aprobado el EIA y durante toda la vigencia de la certificación ambiental, en este orden de ideas, y considerando lo establecido en el artículo 28, creemos que la actualización también puede presentarse en este mismo espacio de tiempo, siempre que se haya producido un cambio en el EIA.

La actualización, tal como lo regula el Reglamento del SEIA, constituye una obligación del titular de un EIA, la cual responde a la necesidad de "actualizar" los planes que contienen los EIA, los cuales quedarían desfazados en dos supuestos, a saber: i) cuando se modifica el EIA o ii) cuando las exigencias y estándares ambientales normativos que fueron considerados al momento de elaborar el EIA han sido cambiados.

Por su parte, el artículo 30, añade una obligación adicional al titular del proyecto, la cual consiste en revisar cada 5 años si alguno de los componentes (planes) de su EIA requiere ser actualizado.

Un aspecto importante, que no debe pasar desapercibido, es que en el artículo 30 se establece que el titular debe presentar la actualización para que la autoridad "la procese" con fines de vigilancia y control de los compromisos ambientales asumidos.

Consideramos que, tanto el artículo 28 como el 30 regulan la actualización de EIA como una actividad de mera sistematización y puesta a punto del estudio, en concreto de los planes que componen la estrategia ambiental, limitando la función de la autoridad a una mera actividad de "procesamiento con fines de vigilancia y control de compromisos ambientales," lo que puede llevarnos a concluir que en la actualización del EIA no cabe actividad de evaluación o reexamen de impactos ambientales.

Ahora bien, el desarrollo que realizan los reglamentos ambientales sectoriales de la Actualización ha sido en algunos casos neutro, es decir no han deducido nuevas reglas particulares a partir de las antes reseñadas reglas generales, y en otros, disruptivo, al haber creado un nuevo enfoque y finalidad para la Actualización.

Así, según este nuevo enfoque, la actualización busca optimizar la eficacia de las medidas de manejo ambiental, a partir de un esfuerzo constante para, a la luz de su aplicación concreta en la realidad, mejorarlas continuamente. 
El primer reglamento ambiental sectorial en regular la actualización según el enfoque de optimización o mejora continua fue el minero23, en el año 2014. Al respecto, su artículo 128 establece:

(...)

La actualización comprende: el análisis de los impactos reales de la operación en curso en los recursos agua, aire, suelo, fauna y flora y otros aspectos ambientales y sociales, contenidos en el estudio, sobre la base de los reportes de monitoreo $\mathrm{u}$ otra fuente de información, a fin que de ser necesario, se propongan mejoras en la estrategia de manejo ambiental aprobada ${ }^{24}$.

$(\ldots)$

Tres años después, por Resolución Ministerial N 056-2017-MINAM, se publicó el Proyecto de norma que aprobaría las “Disposiciones para la Actualización y Modificación de EA para la Mejora Continua de los Proyectos de Inversión Sujetos al SEIA", el cual en su artículo 26 establecía que:

"La actualización del estudio ambiental aprobado es un mecanismo que aplica después de la ejecución del proyecto de inversión, cuya finalidad es la mejora continua de la gestión ambiental de los proyectos de inversión sujetos al SEIA, contribuyendo a la mejora del desempeño ambiental del titular del proyecto de inversión ${ }^{25 "}$.

Si bien este proyecto hasta ahora no se convirtió en regulación legal, la reglamentación de la Actualización como un mecanismo de optimización y mejora continua de los planes de manejo fue recogida, además de en el sector minero, en el sector Transportes ${ }^{26}$, y Pesca y Acuicultura ${ }^{27}$. Otros reglamentos, como el del sector Agrario 28 , Hidrocarburos ${ }^{29}$ o Industria Manufacturera ${ }^{30}$, tratan la Actualización en consonancia con la Ley y Reglamento del SEIA; en el caso del

23 Decreto Supremo No 040-2014-EM. Reglamento de Protección y Gestión Ambiental para las Actividades de Explotación, Beneficio, Labor General, Transporte y Almacenamiento Minero, Diario Oficial El Peruano. Lima, Perú. 25 de setiembre de 2014.

24 Ídem.

25 Resolución Ministerial N 056-2017-MINAM. Disponen la prepublicación del Proyecto de Decreto Supremo que aprueba las “Disposiciones para la Actualización y Modificación de Estudios Ambientales para la Mejora Continua de los Proyectos de Inversión Sujetos al Sistema Nacional de Evaluación de Impacto Ambiental (SEIA)”. Diario Oficial El Peruano. Lima, Perú. 24 de febrero de 2017.

26 Decreto Supremo N 004-2017-MTC. Reglamento de Protección Ambiental para el Sector Transportes. Diario Oficial El Peruano. Lima, Perú. 17 de febrero de 2017. Artículo 19.

27 Decreto Supremo Nº12-2019-PRODUCE. Reglamento de Gestión Ambiental de los Subsectores Pesca y Acuicultura. Diario Oficial El Peruano. Lima, Perú. 11 de agosto de 2019.

28 Decreto Supremo N 019-2012-AG. Reglamento de Gestión Ambiental del Sector Agrario. Diario Oficial El Peruano. Lima, Perú. 14 de noviembre de 2012.

29 Decreto Supremo N 039-2014-EM. Reglamento para la Protección Ambiental en las Actividades de Hidrocarburos. Diario Oficial El Peruano. Lima, Perú. 12 de noviembre de 2014.

30 Decreto Supremo N 017-2015-PRODUCE. Reglamento de Gestión Ambiental para la Industria Manufacturera y Comercio Interno. Diario Oficial El Peruano. Lima, Perú. 6 de junio de 2015. 
sector Vivienda31, el tratamiento es original, debido a que contempla la Actualización en caso durante la supervisión estatal se determine la insuficiencia del plan de manejo ambiental.

La necesidad de mejorar o adecuar constantemente los planes de manejo ambiental es común a todos los EA, y es por ello que la regulación aplicable debería seguir criterios básicos uniformes, reservando a los sectores la definición de cuestiones particulares, propias de la especialidad de las actividades económicas que tienen bajo su competencia.

Finalmente, si bien los términos de la actualización vigente se han establecido por ahora para casos de instrumentos preventivos, lo cierto es que la actualización de los instrumentos correctivos es una necesidad también, por ello la regulación sectorial la prevé, como lo veremos más adelante, para el sector hidrocarburos y el subsector pesquero ${ }^{32}$.

\section{b) La Modificación}

Ningún otro instrumento pone de manifiesto tan claramente la inflexibilidad del sistema estimativo vigente como lo hace la modificación de instrumentos ambientales. En primer término, la legislación general solo la prevé para casos de instrumentos preventivos, haciendo al margen a los instrumentos correctivos. Este problema también es abordado por los reglamentos sectoriales, los cuales habilitan la posibilidad de modificar instrumentos correctivos, sin embargo, no queremos dejar de reforzar la premisa -dentro de un esquema de estimación ambiental- de que no se justifica que no se regule en las normas transectoriales generales la oportunidad de modificar un instrumento correctivo.

Adicionalmente a este hecho, se suele criticar la velocidad con la que la autoridad evalúa los instrumentos de modificación, los cuales, al resultar esencialmente preventivos, condicionan la implementación de nuevos componentes al interior de los proyectos, los cuales enfrentan urgencias sociales y financieras. Como respuesta a este esquema de modificación, se instauró, por norma de la PCM, el régimen de ITS, reservado para impactos no significativos, sin embargo, subsisten reglas sectoriales disímiles en cuanto a su aplicación, a saber: i) respecto del número máximo de ITS para plantear por cada proyecto, ii) respecto a qué tipo de componentes/procesos considerar como generadores de impactos "no significativos", iii) respecto a las condiciones ecosistémicas que restringen la presentación de un ITS (como plantear la incorporación de componentes dentro de ecosistemas frágiles), entre otras.

31 Reglamento de Protección Ambiental para proyectos vinculados a las actividades de Vivienda, Urbanismo, Construcción y Saneamiento, Decreto Supremo N015-2012-VIVIENDA, modificado por Decreto Supremo Nº14-2019-VIVIENDA. Diario Oficial El Peruano. Lima, Perú. 3 de abril del 2019.

32 Decreto Supremo N 012-2019-PRODUCE. Reglamento de Gestión Ambiental de los Subsectores Pesca y Acuicultura. Diario Oficial El Peruano. Lima, Perú. 11 de agosto de 2019, Artículo 48. 


\section{ii. Instrumentos de gestión ambiental complementarios}

Al remitirnos a la normativa general nacional, podemos obtener nociones de cómo el legislador trató de enfocar la estimación de impactos dentro del proceso general de evaluación ambiental. Si tomamos como referencia, por ejemplo, el primer artículo de la Ley del Sistema Nacional de Evaluación del Impacto Ambiental (SEIA), publicada en el año 2001, encontraremos remisiones orientadas a un esquema preferentemente preventivo de corrección anticipada:

“La presente Ley tiene por finalidad:

a) La creación del Sistema Nacional de Evaluación del Impacto Ambiental (SEIA), como un sistema único y coordinado de identificación, prevención, supervisión, control y corrección anticipada de los impactos ambientales negativos derivados de las acciones humanas expresadas por medio del proyecto de inversión ${ }^{33 "}$.

¿Y qué se debe considerar como parte de lo preventivo? Toda aquella medida orientada a la estimación de impactos de manera previa a su materialización, es decir, el momento que consideró oportuno el legislador para realizar la estimación ambiental de los proyectos adscritos al SEIA fue aquel en donde los impactos se habían previsto, pero no materializado.

En el año 2005, con la publicación de la Ley General del Ambiente, se incluyó un amplio espectro de herramientas orientadas al cumplimiento de objetivos y metas ambientales a nivel nacional, a través de los instrumentos de gestión ambiental, regulados en el artículo 17:

“17.1 Los instrumentos de gestión ambiental podrán ser de planificación, promoción, prevención, control, corrección, información, financiamiento, participación, fiscalización, entre otros, rigiéndose por sus normas legales respectivas y los principios contenidos en la presente Ley 34 ".

Gracias a esta inclusión, la legislación ambiental nacional permitió reconocer mecanismos adicionales a los preventivos ya previstos por la normativa del SEIA. Sin embargo, no fue sino hasta la publicación del Reglamento de la Ley del SEIA (año 2009), que el sistema los acogió formalmente, bajo el criterio de complementariedad, así el artículo 13 de la referida norma prescribe:

"Los instrumentos de gestión ambiental no comprendidos en el SEIA son considerados instrumentos complementarios al mismo. Las obligaciones que se establezcan en dichos instrumentos deben ser determinadas de forma concordante con los objetivos, principios y criterios que se señalan en la Ley y el presente Reglamento, bajo un enfoque de integralidad y

33 Ley N²7446. Ley del Sistema Nacional de Evaluación del Impacto Ambiental. Diario Oficial El Peruano. Lima, Perú. 23 de abril de 2001, Artículo 1.

34 Ley N²8611. Ley General del Ambiente. Diario Oficial El Peruano. Lima, Perú. 15 de octubre de 2015. 
complementariedad de tal forma que se adopten medidas eficaces para proteger y mejorar la salud de las personas, la calidad ambiental, conservar la diversidad biológica y propiciar el desarrollo sostenible, en sus múltiples dimensiones ${ }^{35}$.

De este modo, y luego de analizar la aplicación conjunta de los artículos antes citados, no debería quedar duda alguna de que, si bien el SEIA privilegia la estimación ambiental enfocada en instrumentos preventivos, también el esquema correctivo (y sus instrumentos) son reconocidos como complementarios al sistema, por lo que deben regirse por reglas similares a las que se aplican a sus instrumentos principales, incluyendo los criterios de estimación que deben formar parte de la normativa ambiental general.

Pero entonces, ¿a qué nos referimos al mencionar este "esquema correctivo"? Hasta el momento, la premisa que habíamos manejado en líneas generales es que la estimación resulta crucial para la previsión de proyectos futuros y para la corrección adaptativa del proyecto al entorno ¿Y qué pasaría si el proyecto sí existiese a la fecha de la estimación? Para este caso particular, la estimación resulta igualmente necesaria, aunque diversa.

Siguiendo este razonamiento, el esquema correctivo no es sino aquel destinado a aplicarse para aquellas actividades en curso, que requieren adaptarse al régimen ambiental vigente. ¿Y cómo se materializa este esquema correctivo? Consideramos que existen dos supuestos bajo los cuales se implementa los esquemas de corrección: i) la incorporación de nuevas actividades al SEIA y ii) la regularización de componentes no evaluados de proyectos en marcha.

Respecto del primero, el régimen de corrección intenta responder a la necesidad de incorporar progresivamente -y por primera vez- una serie de actividades económicas dentro del esquema normativo de gestión de impactos ambientales. Así, por ejemplo, el primer reconocimiento ambiental formal y sectorial que se estableció para las actividades minero-metalúrgicas en el Perú, fue impulsado por el Decreto Supremo N 016-93-EM, el cual aprobó el Reglamento del Título Décimo Quinto del Texto Único Ordenado de la Ley General de Minería, sobre el medio ambiente. Un caso más reciente fue el del sector Comercio Interno, el cual fue incorporado al SEIA, a través del Reglamento de Gestión Ambiental para la Industria Manufacturera y Comercio Interno ${ }^{36}$.

Para ambos casos, tanto el régimen de Minería como el de Comercio Interno, establecieron la posibilidad de que las actividades en curso (a la fecha de entrada en vigencia de cada norma) adscritas a cada sector económico, pudieran adecuarse e incorporarse al esquema de cumplimiento ambiental, mediante el trámite e

35 Decreto Supremo N 019-2009-MINAM. Reglamento del Sistema Nacional de Evaluación del Impacto Ambiental. Diario Oficial El Peruano. Lima, Perú. 25 de setiembre de 2009.

36 Decreto Supremo N 017-2015-PRODUCE. Reglamento de Gestión Ambiental para la Industria Manufacturera y Comercio Interno. Diario Oficial El Peruano. Lima, Perú. 6 de junio de 2015. 
implementación de un instrumento correctivo específico: el Programa de Adecuación y Manejo Ambiental - PAMA.

Por otro lado, respecto del segundo supuesto, la normativa sectorial vigente también prevé situaciones excepcionales para aquellos casos en que los titulares de proyectos de inversión fueron incorporando progresivamente -durante la vida útil del proyecto- componentes o procedimientos nuevos que debieron, pero no fueron evaluados en el marco del SEIA. En función a ello, se estableció la aplicación de instrumentos de naturaleza extraordinaria que abrían la posibilidad de regularizar los componentes no evaluados, mediante la presentación de instrumentos extraordinarios de adecuación. Un ejemplo de esto, se puede observar con la creación del denominado Plan Ambiental Detallado - PAD ${ }^{37}$, del subsector Hidrocarburos, el cual tiene como finalidad "regularizar", o mejor dicho, incorporar componentes no evaluados a los instrumentos de gestión ambiental del proyecto.

Vayamos a un ejemplo para el primer caso (instrumentos correctivos): una planta de fabricación de harina de pescado, con operaciones iniciadas antes de la vigencia del SEIA, debe adecuarse a las nuevas normas ambientales. El proceso de adecuación implica corregir los impactos reales que viene ocasionando, por lo tanto, la estimación ambiental ya no se enfocará en la identificación de potenciales impactos, sino que deberá analizar la eficacia teórica de las medidas de mitigación en el entorno social y ambiental.

El evaluador deberá responderse preguntas tales como ¿la medida de mitigación prevista para el impacto (concreto y real) " $\mathrm{A}$ " es suficiente para restablecer la calidad de componente ambiental " $\mathrm{B}$ " ? ¿la medida " $\mathrm{C}$ " garantizar la rehabilitación de los procesos ecosistémicos comprometidos con la operación? ¿en cuánto tiempo se restablecerá lo dañado? ¿cuál es la percepción ciudadana respecto de la actividad y cómo las medidas de manejo ambiental las afectan?

Como podemos apreciar, la estimación ambiental no es una herramienta exclusiva para ser aplicada a los instrumentos preventivos, también debe ser empleada para los correctivos, aunque de diversa forma.

Igualmente, para el caso de los instrumentos extraordinarios de regularización de componentes no declarados, como el PAD del sector Hidrocarburos, la lógica es similar a la expuesta para los instrumentos correctivos, se trata de evaluar componentes del proyecto que no fueron evaluados en su momento y por lo tanto, implican una evaluación estimativa integral, es decir, analizar cómo el nuevo componentes interactúa con el proyecto en su conjunto, y si las medidas de mitigación primigenias siguen siendo suficientes en el nuevo escenario que plantea el o los nuevos componentes.

37 Decreto Supremo No 023-2018-EM. Modifica el Reglamento de Protección Ambiental en las actividades de Hidrocarburos. Diario Oficial El Peruano. Lima, Perú. 7 de setiembre de 2018. 
Una serie de interrogantes adicionales se nos presentan en torno a este esquema de instrumentos complementarios ¿La estimación, dentro de los instrumentos correctivos, debe terminar respondiendo únicamente al contexto de adecuación? La respuesta vendría a ser negativa, ya que, después de todo, además de la estimación de los impactos actuales de los proyectos en marcha, también se requiere cierto nivel de estimación enfocada para aquellos cambios eventuales que puedan darse a nivel del proyecto durante toda su vida útil.

La manifestación más concreta de este doble factor estimativo se pone de manifiesto al observar cómo regula el régimen ambiental de industria el instrumento denominado Diagnóstico Ambiental Preliminar - DAP. Mediante la Décimo Segunda Disposición Complementaria Final de su reglamento, se reconoce a la DAP como un instrumento correctivo, pero además se abre la posibilidad de estimar los impactos futuros, mediante la presentación de modificaciones o actualizaciones del Plan de Manejo Ambiental de la referida DAP, conforme a lo siguiente:

“Los Diagnósticos Ambientales Preliminares (DAP) aprobados antes de la entrada en vigencia del presente Reglamento, son considerados instrumentos de gestión ambiental de tipo correctivos.

La actualización y modificación del Plan de Manejo Ambiental del DAP, se realizará según lo establecido en el presente Reglamento para los instrumentos de gestión ambiental de tipo correctivo. El titular podrá solicitar a la autoridad competente, la actualización del Plan de Manejo Ambiental en los componentes que lo requieran (... $)^{38 "}$.

En ese sentido, el legislador reconoce la posibilidad de modificar a futuro el instrumento correctivo, a modo de respuesta a la realidad variable del proyecto de inversión; y, respecto de la mención que realiza a la actualización, nos preguntamos ¿Se permitirá en el sector industria utilizar la actualización con fines de optimización y mejora continua de las medidas de manejo ambiental o solo con fines de sistematización?

\section{A manera de conclusión}

A 20 años de la aprobación, por insistencia del Congreso, de la Ley del SEIA y a 11 años de la vigencia efectiva del SEIA, hemos querido ofrecer una reflexión acotada a temas que creemos deberían formar parte de un análisis mayor que involucre a todo el SEIA, y que identifique sus aciertos, que los tiene y muchos, pero también sus oportunidades de mejora.

El SEIA produce soluciones a problemas especiales y complejos, que se manifiestan como EIA aprobados, por lo tanto, no debe olvidarse jamás que la

38 Decreto Supremo N 017-2015-PRODUCE. Reglamento de Gestión Ambiental para la Industria Manufacturera y Comercio Interno. Diario Oficial El Peruano. Lima, Perú. 6 de junio de 2015.

Décimo Segunda Disposición Complementaria Final. 
solución, para ser eficaz, debe no solo ser la idónea sino también haber sido construida con quienes padecen el problema. Para el caso que nos ocupa, la solución (EA) sin la participación de los involucrados puede ser percibida como imposición.

Creemos que los esfuerzos de las autoridades públicas a que se refiere el Acuerdo de Escazú, no se limitan a la actividad de evaluación de los estudios ambientales, sino a una actividad especial, a cargo de los entes públicos, paralela e integrada a la que realiza el proponente del proyecto, encaminada a asegurar la participación de la población y la calidad de estudio de línea de base.

Esta actividad de los funcionarios públicos, encaminada a promover y lograr el involucramiento, crítico y constructivo de la población e identificar los elementos clave del estudio de línea de base, tales como área del estudio y los factores ambientales y sociales críticos, no se encuentra suficientemente presente en la regulación transectorial del SEIA.

En ese sentido, urge completar nuestra regulación transectorial con normas que establezcan una participación más activa, constante y cercana de los funcionarios públicos, que deje atrás el enfoque pasivo, normativo y evaluador y lo complemente con otro, que ubique al Estado (o su representante) en el campo y como un actor relevante del proceso de elaboración del EA.

El acto administrativo por el cual se aprueba el EIA se denomina "certificación ambiental", denominación que alude a una acción del Estado, la cual no puede realizarse sin un involucramiento cercano al proponente, al entorno y a la población, desde faces iniciales de la elaboración del EIA.

Creemos fundamental que el Estado participe activamente:

i. Capacitando y sensibilizando a la población.

ii. Acompañando la delimitación del área de estudio de la línea base, en función a una caracterización preliminar del entorno social, institucional y ambiental del proyecto, que implica la identificación de los actores sociales e institucionales, teniendo en consideración la identificación preliminar de los impactos ambientales.

iii. En la aproximación con los actores sociales e institucionales, para conocer, de forma temprana y directa sus percepciones, intereses, dudas, observaciones y preconceptos relacionados con el proyecto.

Pretender que la certificación ambiental sea creíble, sin un involucramiento temprano, activo y sostenido del Estado, resulta ser una ingenuidad muy costosa para todos los involucrados en el proceso.

Estamos convencidos que, sin un involucramiento efectivo y cercano del Estado en la elaboración de la Línea de Base y la participación ciudadana, el EA no 
recogerá la información suficiente para poder ser un estudio específico y a la medida de lo que exige el entorno socio ambiental con el que se interrelacionará el proyecto.

Los esfuerzos por simplificar y acelerar los procesos administrativos de evaluación de los EA, que se traducen en: i) reducir los plazos de que disponen los organismos públicos para efectuar sus análisis y evaluaciones, ii) introducir figuras como la clasificación anticipada de proyectos similares -asociada a la aprobación de términos de referencia comunes- $y$, iii) el uso compartido de la línea de base ambiental; podrían contribuir en algo, aunque no se ha comprobado que lo hagan, a producir EIA "en masa" y estandarizados. Sin embargo, creemos que la especialidad de los EA es amenazada más intensamente cuando el Estado se repliega en sus oficinas, omite realizar el acompañamiento en campo a la consultora ambiental y proponente, y no promueve activamente la participación ciudadana.

El SEIA debe convertirse en un sistema de identificación, prevención, supervisión, control y corrección de los impactos ambientales derivados de proyectos de inversión, durante todo su ciclo de vida, el cual inicia con su formulación a nivel de factibilidad y concluye con el cierre de las actividades económicas.

Para tal fin es necesario, incorporar en el nivel regulatorio transectorial del SEIA, normas que disciplinen la optimización y mejora adaptativa de los planes ambientales contenidos en instrumentos de gestión ambiental, sean estos preventivos, correctivos o correctivos extraordinarios o de cierre.

Para tal fin, es importante abordar la discusión sobre: cómo sistematizar los IGA, cómo integrarlos en uno solo en caso existan varios IGA para un mismo proyecto, cómo introducir mejoras en los planes ambientales o nuevos estándares o exigencias ambientales; qué lineamientos debe seguir la estimación ambiental cuando se aplica a instrumentos complementarios, qué necesidades satisface la actualización y la modificación de los EA, cuáles son sus diferencias y qué involucran sus procedimientos, etc.

Al parecer, es momento de ponernos a conversar sobre el SEIA. 